\title{
Plaque Quantification by Coronary CT and Intravascular Ultrasound Identifies a Low CT Density Core as a Marker of Plaque Instability in Acute Coronary Syndromes
}

\author{
Theodora BenedeK, ${ }^{1} \mathrm{MD}$, Beata JAKO, ${ }^{2} \mathrm{MD}$, and Imre BenedeK, ${ }^{1} \mathrm{MD}$
}

\begin{abstract}
SUMMARY
The purpose of this study was to demonstrate the relationship between the presence and amount of a low-density core (LDC) with a CT density < 30 Hounsfield units (HU) by coronary computed tomography angiography (CCTA) and IVUS-derived markers of vulnerability in the culprit lesions (CL) of patients with acute coronary syndromes (ACS).

In 43 patients with ACS, 105 coronary plaques were scanned using CCTA and IVUS for the quantitative and qualitative assessment of vulnerability markers.

The presence of a low attenuation plaque (LAP) was identified in $67.4 \%$ of the CL and $29.03 \%$ of the non-CL $(P=$ 0.0001). The presence of a LDC $>6.0 \mathrm{~mm}^{3}$ was significantly correlated with the percentage of the necrotic core (NC) $(22.08 \%$ versus $7.97 \%, P=0.001)$ and the fibro-fatty tissue by IVUS $(18.68 \%$ versus $15.87 \%, P=0.02)$. LDC volumes showed a good correlation with the percentage of the NC $(r=0.7303, P<0.0001)$ and the fibro-fatty tissue in the CL $(r$ $=0.4928, P<0.0008)$. Quantitative plaque analysis revealed a significant difference in plaque composition between CL and non-CL in regards to the LDC $(18.45$ versus $6.5, P<0.001)$, the percentage of NC $(20.74$ versus $18.74, P=0.02)$, fibro-fatty tissue (17.77 versus $15.48, P=0.002)$, and fibrotic tissue (51.68 versus $54.8, P=0.01$ ).

VH-IVUS and CCTA plaque quantification showed that the presence of a low-density $(<30 \mathrm{HU})$ core within the CL of patients with ACS represents a marker of vulnerability and correlates well with other CCTA and IVUS-derived features of vulnerability, particularly the NC of the plaque. (Int Heart J 2014; 55: 22-28)
\end{abstract}

Key words: Vulnerable plaques

$\mathrm{T}$ he detection of vulnerable plaques is one of the most challenging tasks made possible by the recent developments in cardiovascular imaging technologies. Taking into consideration the significant risk associated with vulnerable plaques, which are prone to rupture and rapid evolution towards the development of acute coronary syndromes (ACS), ${ }^{1)}$ the ability to detect features that characterize unstable plaques is of extreme importance. If rupture-prone plaques could be identified in time, the appropriate initiation of adequate therapeutic measures could prevent the evolution to an acute coronary event. $^{2)}$

A vulnerable plaque is characterized by a large necrotic core, a thin fibrous cap demonstrating macrophage infiltration, a large lipid pool, and several specific features such as positive remodeling (PR) or spotty calcifications $(\mathrm{SC}) .{ }^{3)}$ When these characteristics are present, the fibrous cap can rupture and the lipid core, which is thrombogenic, is then exposed to blood flow, inducing thrombus formation and the development of ACS. ${ }^{4}$

The morphological characteristics associated with unstable plaques are generally evaluated using three main imaging methods: Intravascular ultrasound with virtual histology (VH-
IVUS), coronary computed tomography angiography (CCTA), and optical coherence tomography (OCT).

Intravascular ultrasound is currently the gold standard for the detection and assessment of vulnerable plaques due to its ability, when associated with virtual histology, to differentiate the soft atheroma with a lipid reach or necrotic core, which is typically associated with vulnerable plaques, from a fibrous or calcified atheroma, which is generally associated with stable plaques. ${ }^{6}$ VH-IVUS is able to combine intracoronary imaging data with a color-coded representation of plaque components, which are classified as fibrotic, fibro-fatty, calcified, or necrotic core, while at the same time offering the possibility for precise quantification of these components.

CCTA is another imaging technique that can identify specific parameters associated with plaque vulnerability, such as $\mathrm{PR}, \mathrm{SC}$, and burden with low attenuation plaque (LAP). It has been shown that a PR on CCTA is associated with higher percentages of necrotic cores within the plaque on IVUS and that the percentage of the necrotic core by IVUS is significantly higher in plaques with SC identifiable by CCTA compared to non-calcific plaques. ${ }^{8,9)}$ However, although these studies have demonstrated an association between the presence of different

From the ${ }^{1}$ Department of Internal Medicine, University of Medicine and Pharmacy of Tîrgu Mures and ${ }^{2}$ Cardiomed Medical Center, Tîrgu Mures, Romania.

This work was partly supported by the Sectorial Operation al Programme Human Resources Development (SOP HRD), financed from the European Social Fund and by the Romanian Government under the contract number POSDRU 80641.

Address for correspondence: Theodora Benedek, MD, Department of Internal Medicine, University of Medicine and Pharmacy of Tîrgu Mures, Gheorghe Marinescu street no.50, Tîrgu Mures 540136, Romania. E-mail: hintea_teodora@yahoo.com

Received for publication July 23, 2013.

Revised and accepted August 26, 2013.

Released advance online J-STAGE January 27, 2014.

All rights are reserved to the International Heart Journal Association. 
CT and IVUS features of coronary plaques in different clinical settings, the precise correspondence between plaque components classified on the basis of CT attenuations and VH-IVUS derived components, has not yet been clearly established.

Available software programs have enabled CCTA to be used for quantitative analysis of plaque components based on different CT attenuations within the plaque. ${ }^{10)}$ In most ACS cases, CCTA plaque quantification demonstrates a mixed composition of the coronary plaques, containing variable proportions of a lipid-reach core with a low CT density (with mean attenuation values reported in a range between 11 and 99 Hounsfield units (HU)), a fibrous component with higher CT densities (with mean attenuation values reported in a range between 77 and $121 \mathrm{HU}$ ), and calcium. ${ }^{11-13)}$

While many studies published on the topic of CT plaque characterization have demonstrated the presence of a low-density core (LDC) containing lipid-reach atheroma in the culprit lesions, ${ }^{14,15)}$ quantitative assessment of this LDC is almost totally lacking in all these studies. We previously reported that unstable plaques are characterized by larger volumes of lowdensity lipid-reach cores and that a critical volume of $6.0 \mathrm{~mm}^{3}$ plaque with CT density $<30 \mathrm{HU}$ could differentiate between culprit and non-culprit lesions in patients with ACS, with a sensitivity of $100 \%$ and a specificity of $65.8 \%$ (area under the curve 0.89). ${ }^{16)}$ In our previous study, culprit lesions were significantly associated with LDC volumes larger than $6.0 \mathrm{~mm}^{3}$ in unstable patients.

Based on this observation, the aim of the current study was to demonstrate the relationship between the presence and the amount of the LDC (defined as a plaque component with a CT density < $30 \mathrm{HU}$ ) and IVUS-derived markers of vulnerability (eg, the presence of necrotic core, high content in fibrofatty atheroma and PR) in the culprit lesions of patients with ACS. At the same time, we aimed to study the correlation between LDC volume and well established IVUS and CCTA vulnerability features associated with culprit and non-culprit lesions in unstable patients.

\section{MethodS}

Patients: This was a single center prospective non-randomized study to evaluate the CCTA characteristics of coronary lesions compared with the IVUS-derived features of these lesions in patients with ACS.

Forty-three patients with ACS (unstable angina or non-ST acute myocardial infarction) at clinical presentation were enrolled in the study, and each subject underwent 64 multi-slice CT followed by coronary angiography and intravascular ultrasound with virtual histology within 24 hours.

The inclusion criteria were age $>18$ years, documented UA or NSTEMI, and willingness to participate in the study. Patients with contraindications for the repeated administration of contrast agents, an unstable hemodynamic condition, cardiac arrhythmia that precluded a good CT image quality, prior coronary artery bypass grafting, or coronary stenting were excluded from the study.

Patients with ST segment elevation (in whom revascularization should be performed as soon as possible), as well as high-risk patients with an unstable hemodynamic condition, in whom emergent revascularization was indicated without any delay, were excluded for ethical reasons. As European guidelines indicate that an invasive strategy within 24 hours appears to be the reasonable treatment for patients with low or intermediary risk UA/NSTEMI, ${ }^{17)}$ we performed the CT acquisition in the first 6 hours after the presentation, followed immediately by invasive coronary angiography and revascularization, within this timeframe of the recommended 24 hours.

All ethical issues (such as appropriate patient selection according to guidelines, appropriate timeframe for performing cardio CT followed by coronary angiography and IVUS, radiation dosage) were included in the study protocol which was approved by the ethics committee of the center where the study was conducted. Also, all these issues together with the safety issues were carefully explained to the patients selected for this study and all of them gave written informed consent.

Culprit lesions were identified according to the localization of the echocardiographic or electrocardiographic abnormalities correlated with the angiographic appearance of the lesion.

CT analysis: All CT acquisitions were made using a multislice 64 Somatom Sensation CT (Siemens, Germany) with a $64 \times 0.5 \mathrm{~mm}$ detector collimation. During an inspiratory breath-hold, $60 \mathrm{~mL}$ of an iodinated contrast agent (Iopamidol, $370 \mathrm{mg} \mathrm{I} / \mathrm{mL}$, Bayer Healthcare, Germany) was infused at a speed of $4.0 \mathrm{~mL} /$ second followed by $20 \mathrm{~mL}$ at $2.0 \mathrm{~mL} / \mathrm{second}$. All examinations were preceded by the administration of a short-acting beta-blocker to achieve the desired heart rate and were conducted only after achieving a stable heart rate below 60 beats/minute. The extent of calcium in the coronary arteries was assessed during the precontrast scans taken at the start of the imaging. If this calculated calcium score was higher than 1.000 , the patient was excluded from the study because it is considered that a calcium score $>1.000$ precludes technically adequate $\mathrm{CT}$ coronarography due to intense calcifications.

After optimization of scanning protocols, the total amount of radiation exposure for undergoing coronary $\mathrm{CT}$ ranged between 11 and $14 \mathrm{mSv}$ per case (mean $12.6 \mathrm{mSv}$ ), doses for which the life-time attributable risk is considered to be very small. The mean radiation exposure for undergoing invasive coronary angiography and PCI was $7.8 \mathrm{mSv}$, which added to the $12.6 \mathrm{mSv}$ recorded for cardio CT represents a total of 20.4 $\mathrm{mSv}$ mean radiation dose per case, which is within the generally accepted range of radiation in cardiology imaging and intervention. ${ }^{18)}$ For instance, the amount of radiation exposure related to a radionuclide isotope myocardial perfusion imaging could reach $29 \mathrm{mSv}$, much higher than the total dose of radiation recorded in our study.

All acquired images were transferred to a workstation (Siemens, Germany) for data processing, measurements, and interpretation. Based on recommendations by the American Heart Association, the coronary tree of each patient was divided into 17 segments. ${ }^{19)}$ Therefore, in the 43 patients, 731 coronary segments were scanned for the presence and analysis of coronary plaques, and each plaque was analyzed in cross-sectional, longitudinal, and multiplanar reformation images.

Qualitative plaque analysis included the presence and assessment of vessel remodeling, spotty calcification, and low attenuation core. Plaque quantification included the determination of plaque volume and the extent of low density core within the plaque. The cutoff point of $30 \mathrm{HU}$ for defining the LDC was manually selected, and the LDC volume and percentage 
from the total plaque volume were calculated.

VH-IVUS analysis: IVUS assessment was performed using S5 InVision Gold intravascular ultrasound equipment. Coronary ultrasound catheters were introduced into the coronary artery distal to the lesion and were retracted at a speed of $1 \mathrm{~mm} /$ sec using an automatic tracking device. After aquisition of the IVUS videoloops, coronary plaques were manually traced in cross-sectional images and then processed using the virtual images superimposed on the frames aquired.

The VH-IVUS assessment included the following:

-assessment of the mean lumen area at the site of maximum stenosis

-calculation of the remodeling index

-plaque quantification at the site of maximum stenosis, including determination of percentages of fibro-fatty tissue, necrotic core, fibrotic tissue, and calcified plaque.

Definitions for plaque characterization: The remodeling index (RI) as obtained by CT was expressed as the ratio between the vessel diameter at the site of the lesion and the vessel diameter at the reference segment located proximal to the lesion.

The RI as obtained by IVUS was defined using the following formula: [External elastic membrane cross-sectional area (EEM CSA) measured at the narrowest lesion site-reference EEM CSA]/reference EEM CSA × 100 .

Plaques were classified as having positive remodeling

(PR) when the RI at the plaque site was $>1.1$.

$\mathrm{SC}$ was defined as calcification $<3 \mathrm{~mm}$ in size.

The LDC within a coronary plaque was defined as the plaque component with a CT density below $30 \mathrm{HU}$.

The LAP was defined as the plaque containing a LDC larger than $6.0 \mathrm{~mm}^{3}$.

The primary objective of this study was to demonstrate the association between the presence of LAP classified by CCTA and the markers of vulnerability determined by IVUS (PR, high percentage of necrotic core or fibro-fatty atheroma) in culprit lesions of patients with ACS.

The secondary objectives were as follows:

a. To demonstrate the correlation between increased volumes of CCTA-determined LDC and the amount of VHIVUS-derived vulnerable components within the plaque (NC and fibro-fatty atheroma) in culprit lesions

b. To study the correlation between LDC volume and well-established IVUS and CCTA vulnerability features associated with culprit and non-culprit lesions in unstable patients. Statistical analysis: All statistical analyses were performed using Graph Pad InStat software, version 3.1, (GraphPad Software Inc., San Diego, CA, USA). Categorical variables are expressed as percentages. Fisher's exact test was used for comparing the categorical variables. Continuous values are expressed as the mean and standard deviation, and statistical significance was determined using the Mann-Whitney test. Linear regression was used to assess the correlation between LDC volume by CCTA and necrotic core by VH-IVUS. Statistical significance was considered for a $P$ value $<0.05$, and all $P$ values were 2-sided.

\section{RESUlts}

Forty-three patients (29 men, mean age 60.0 years, range 34-76 years) who had undergone CT imaging and invasive coronary angiography with IVUS were enrolled. In total, 105 coronary plaques (43 culprit and 62 non-culprit) were found and analyzed with CCTA and VH-IVUS for quantitative and qualitative assessment of vulnerability markers.

The clinical baseline characteristics of the study population are listed in Table I.

Low CT-attenuation plaques and IVUS-derived markers of vulnerability in culprit lesions: Low CT-attenuation plaques (LAP) were defined as coronary plaques in which the volume of the LDC (with CT attenuation $<30 \mathrm{HU}$ ) was larger than 6.0 $\mathrm{mm}^{3}$.

Based on this classification, we identified the presence of a LAP in $67.4 \%$ of the culprit lesions and $29.0 \%$ of the nonculprit lesions $(P=0.0001)$.

The presence of a LDC $>6.0 \mathrm{~mm}^{3}$, which classified the plaque as a LAP, was significantly correlated with the percentage of the NC and the fibro-fatty tissue by IVUS $(22.1 \%$ versus $7.9 \%, P=0.001$ for $\mathrm{NC}$, and $18.6 \%$ versus $15.8 \%, P=0.02$ for fibro-fatty). In turn, VH-IVUS analysis did not show any significant difference between the fibrotic or calcific tissue in LAP-positive lesions and LAP-negative lesions (50.4\% versus $54.2 \%, P=0.07$ for fibrotic and $8.7 \%$ versus $11.9 \%, P=0.05$ for calcified tissue). Also, the RI according to IVUS and the mean lumen area at the site of maximum stenosis were not significantly different between the LAP-positive plaques and LAP-negative ones (1.2 versus $1.2, P=0.6$ ) (Table II).

LDC volumes and necrotic core within the plaque of culprit lesions: LDC volumes determined by CCTA showed a good correlation with the percentage of the necrotic core at the site of the maximum area stenosis $(r=0.7303, P<0.0001)$ in culprit lesions.

Also, the LDC volumes correlated reasonably well with the percentage of fibro-fatty tissue by VH-IVUS in culprit lesions ( $r=0.4928, P<0.0008)$. At the same time, LDC volume presented an inverse moderate but significant correlation with the amount of fibrotic tissue identified by IVUS ( $r=-0.4679$, $P=0.001)$, as well as with the amount of calcified tissue within the culprit plaques $(r=-0.371, P=0.01)$ (Figure 1).

LDC volume and other features of vulnerability in culprit versus non-culprit lesions: Similar to other well established markers of vulnerability, LDCs were significantly associated with culprit lesions. The presence of a LAP with a LDC > 6.0 $\mathrm{mm}^{3}$ was identified in $67.4 \%$ of culprit lesions and in $29.0 \%$ of

Table I. Baseline Characteristics of Study Population $(n=43)$

\begin{tabular}{lc}
\hline Characteristic & Values $n(\%)$ \\
\hline Age, years & \\
Mean (median) & 60.0 \\
Range & $34-76$ \\
Gender, male & $29(67.4)$ \\
Clinical presentation & \\
$\quad$ Unstable angina (UA) & $22(51.1)$ \\
$\quad$ Non ST elevation myocardial infarction (NSTEMI) & $21(48.8)$ \\
Cardiovascular risk factors & \\
$\quad$ Hypertension & $24(55.8)$ \\
$\quad$ Hyperlipidemia & $19(44.1)$ \\
Diabetes & $7(16.2)$ \\
Smoker* & $24(55.8)$ \\
Obesity (BMS $\left.>25 \mathrm{~km} / \mathrm{m}^{2}\right)$ & $17(39.5)$ \\
\hline
\end{tabular}

* past or present. Data are represented as mean+/-standard deviation or as number (percentage). 
Table II. Low CT-Attenuation Plaques and IVUS-Derived Markers of Vulnerability in Culprit Lesions

\begin{tabular}{|c|c|c|c|}
\hline & $\begin{array}{c}\text { No LAP } \\
(<6.0 \mathrm{~mL} \text { soft plaque } \\
\text { with }<30 \text { HU CT density })\end{array}$ & $\begin{array}{c}\text { LAP } \\
(>6.0 \mathrm{~mL} \text { soft plaque } \\
\text { with }<30 \text { HU CT density })\end{array}$ & $P$ \\
\hline Necrotic core $(\%)$ & & & 0.001 \\
\hline Mean +/- SD & $17.9+/-2.3$ & $22.0+/-4.0$ & \\
\hline $95 \%$ confidence interval & $16.6-19.3$ & $20.5+/-23.6$ & \\
\hline Fibro-fatty tissue $(\%)$ & & & 0.02 \\
\hline Mean +/- SD & $15.8+/-4.2$ & $18.6+/-3.4$ & \\
\hline $95 \%$ confidence interval & $13.4-18.3$ & $17.3-19.9$ & \\
\hline Fibrotic tissue $(\%)$ & & & 0.07 \\
\hline Mean +/- SD & $54.2+/-6.2$ & $50.4+/-6.2$ & \\
\hline $95 \%$ confidence interval & $50.6-57.7$ & $48.1-52.8$ & \\
\hline Calcified tissue $(\%)$ & & & 0.05 \\
\hline Mean +/- SD & $11.9+/-6.8$ & $8.7+/-3.7$ & \\
\hline $95 \%$ confidence interval & $8.0-15.8$ & $7.3-10.2$ & \\
\hline Mean lumen area at the site of maximum stenosis $\left(\mathrm{mm}^{2}\right)$ & & & 0.3 \\
\hline Mean +/- SD & $3.5+/-0.6$ & $3.7+/-0.6$ & \\
\hline $95 \%$ confidence interval & $3.1-3.8$ & $3.4-3.9$ & \\
\hline Remodeling index & & & 0.6 \\
\hline Mean +/- SD & $1.2+/-0.1$ & $1.2+/-0.9$ & \\
\hline $95 \%$ confidence interval & $1.1-1.2$ & $1.1-1.2$ & \\
\hline
\end{tabular}
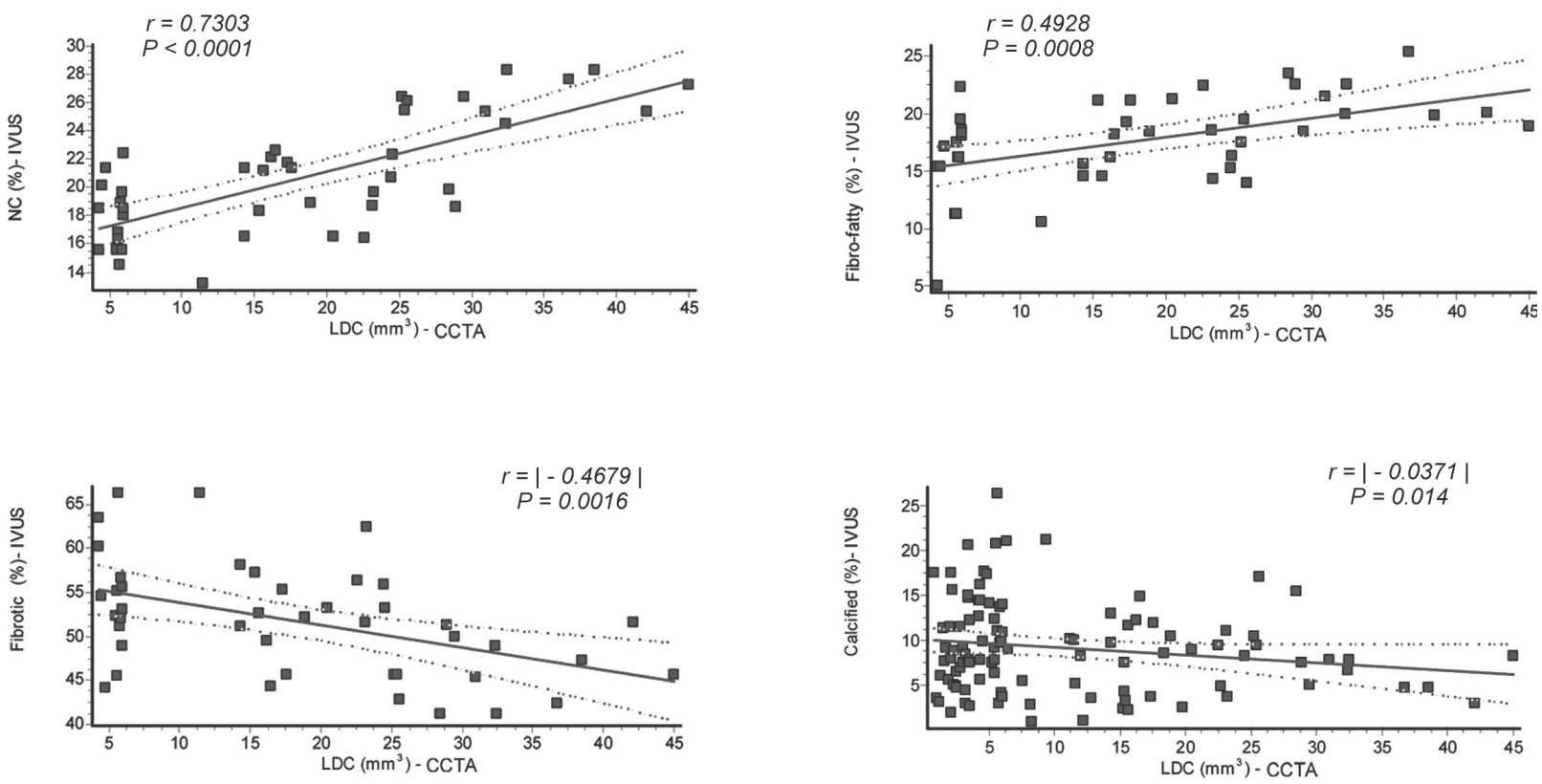

Figure 1. Linear regression for assessment of correlation between volume of LDC and VH-IVUS-derived plaque components.

non-culprit lesions $(P=0.0001)$. Positive remodeling (with calculated RI $>1.1$ ) was present in $86.0 \%$ of the culprit lesions and $24.2 \%$ of the non-culprit ones by CCTA $(P<0.0001)$, and in $81.3 \%$ of culprit lesions and $33.8 \%$ of non-culprit ones by IVUS $(P<0.0001)$. Spotty calcifications were present in $65.1 \%$ of culprit lesions and in $29.0 \%$ of non-culprit ones $(P<$ 0.0001) (Figure 2).

Quantitative plaque analysis using IVUS and CCTA revealed a significant difference in plaque composition in culprit versus non-culprit lesions in regards to LDC $(18.4+/-11.6$ versus $6.5+/-3.4, P<0.001)$, plaque burden $(107.6+/-40.2$ versus $71.0+/-18.7, P<0.0001)$ percentage of necrotic core
$(20.7+/-4.0$ versus $18.7+/-4.4, P=0.02)$, percentage of fibrofatty tissue $(17.7+/-3.9$ versus $15.4+/-3.6, P=0.002)$, and percentage of fibrotic tissue $(51.6+/-6.4$ versus $54.8+/-6.6, P$ $=0.01$ ). However, the amount of calcium within the plaque was not significantly different between culprit lesions as compared with the non-culprit ones. Remodeling index was significantly higher in the culprit lesions, determined using either CCTA $(1.2+/ 0.1$ versus $1.0+/-0.1, P<0.0001)$ or IVUS $(1.2$ $+/-0.1$ versus $1.0+/-0.1, P<0.0001$ ) (Table III).

Interestingly, when comparing the VH-IVUS plaque features in the 3 different types of plaques studied (positive LAP culprit, negative LAP culprit, or no culprit), we observed that 
negative LAP culprit plaques, in which no significant LDC was identified, exhibited a similar composition as non-culprit plaques in regards to NC, fibro-fatty, fibrotic, and calcified tissue (Figure 3). Only the RI remained significantly different between negative LAP culprit plaques and non-culprit plaques (1.2 versus $1.0, P<0.0001)$.

\section{DisCUSSION}

Intracoronary vulnerable plaques are associated with a high risk of plaque rupture and the development of acute coronary syndromes. ${ }^{20)}$ Therefore, the detection of vulnerable plaques represents one of the main goals for the new imaging techniques in cardiology.

The current gold standard for assessing vulnerable

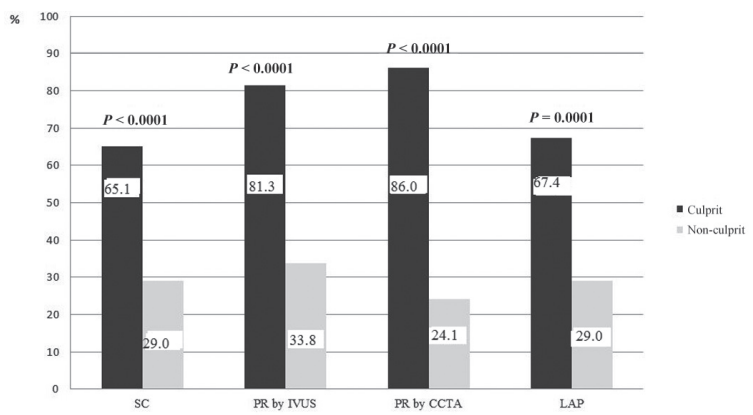

Figure 2. Presence of vulnerability features in culprit versus non-culprit lesions. plaques is intravascular ultrasound associated with virtual histology (IVUS-VH) due to the excellent visualization of intrac-

Table III. IVUS and CCTA Plaque Characteristics in Culprit Versus Nonculprit Lesions

\begin{tabular}{|c|c|c|c|}
\hline & Culprit & Non-culprit & $P$ \\
\hline \multicolumn{4}{|l|}{ CCTA plaque characterization } \\
\hline $\mathrm{LDC}\left(\mathrm{mm}^{3}\right)$ & & & $<0.0001$ \\
\hline Mean +/- SD & $18.4+/-11.6$ & $6.5+/-3.4$ & \\
\hline $95 \%$ confidence interval & $14.8-22.0$ & $5.6-7.5$ & \\
\hline Plaque burden $\left(\mathrm{mm}^{3}\right)$ & & & $<0.0001$ \\
\hline Mean +/- SD & $107.7+/-40.2$ & $71.0+/-18.7$ & \\
\hline $95 \%$ confidence interval & $95.3-120.0$ & $66.2-75.7$ & \\
\hline Remodeling index & & & $<0.0001$ \\
\hline Mean +/- SD & $1.2+/-0.1$ & $1.0+/-0.1$ & \\
\hline $95 \%$ confidence interval & $1.2-1.3$ & $0.9-1.0$ & \\
\hline \multicolumn{4}{|l|}{ IVUS plaque characterization } \\
\hline Necrotic core $(\%)$ & & & 0.02 \\
\hline Mean +/- SD & $20.7+/-4.0$ & $18.7-4.4$ & \\
\hline $95 \%$ confidence interval & $19.4-22.0$ & $17.6-19.8$ & \\
\hline Fibro-fatty tissue $(\%)$ & & & 0.002 \\
\hline Mean +/- SD & $17.7+/-3.9$ & $15.4+/-3.6$ & \\
\hline $95 \%$ confidence interval & $16.5-18.9$ & $14.5-16.4$ & \\
\hline Fibrotic tissue $(\%)$ & & & 0.01 \\
\hline Mean +/- SD & $51.6+/-6.3$ & $54.8+/-6.6$ & \\
\hline $95 \%$ confidence interval & $49.7-53.6$ & $53.1-56.5$ & \\
\hline Calcified tissue $(\%)$ & & & 0.2 \\
\hline Mean +/- SD & $9.8+/-5.1$ & $10.9+/-5.5$ & \\
\hline $95 \%$ confidence interval & $8.2-9.5$ & $9.5-12.3$ & \\
\hline Remodeling index & & & $<0.0001$ \\
\hline Mean +/- SD & $1.2+/-0.1$ & $1.0+/-0.1$ & \\
\hline $95 \%$ confidence interval & $1.1-1.2$ & $1.0-1.1$ & \\
\hline
\end{tabular}

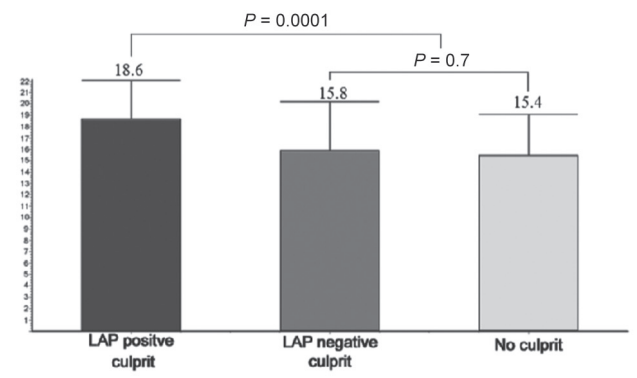

Fibro-fatty tissue (\%)

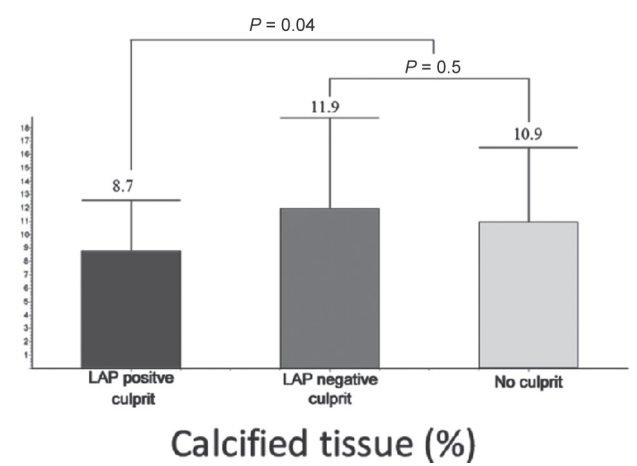

Figure 3. Analysis of VH-IVUS plaque features in the positive LAP culprit, negative LAP culprit and non-culprit plaques showing that NC and fibro-fatty tissue are present in a significantly greater extent in the LAP positive culprit plaques, while negative LAP culprit plaques exhibit a similar composition as non-culprit plaques in regards to NC, fibro-fatty, fibrotic, and calcified tissue. 
oronary plaques and the quantification of plaque components. ${ }^{21,22)}$

However, the discomfort associated with an interventional technique precludes IVUS technology from being used on a large scale for the detection of vulnerable plaques, and noninvasive techniques have emerged to replace interventional imaging techniques for complex assessment of intracoronary plaques. The recent progress in non-invasive imaging techniques, as represented by CCTA, has enabled noninvasive visualization of coronary plaques along with plaque quantification. ${ }^{23)}$

Several studies that compared CCTA with intravascular ultrasound have validated the role of CCTA for the detection of coronary plaques, reporting sensitivities and specificities that vary between 80 and $90 \% .^{24,25)}$ However, CT imaging is not restricted only to plaque visualization, as it provides additional information regarding plaque burden, composition and remodeling, which are directly correlated with plaque vulnerability. ${ }^{26)}$

In a prospective study including 1059 patients who were followed for a mean period of 2.3 years after having undergone CCTA, Motoyama, et al demonstrated that specific plaque parameters such as PR and low CT attenuation may be associated with a particularly high risk for plaque rupture and the development of an acute coronary event. ${ }^{27)}$ Another retrospective study demonstrated that culprit lesions present a more positive remodeling ( 815 versus $12 \%)$, more low-density $(<30 \mathrm{HU})$ plaque components (79\% versus $9 \%)$, and a higher prevalence of spotty calcifications ( $63 \%$ versus $21 \%$ ), and all of these features were shown to represent significant predictors for ACS. ${ }^{28)}$

It has been speculated that the identification of very low CT densities (below $30 \mathrm{HU}$ ) within a plaque may be associated with a higher predisposition towards rupture and could therefore represent a marker of vulnerability. ${ }^{27)}$ In a previous study, based on CCTA analysis without an IVUS control, we reported that the identification of a low density $(<30 \mathrm{HU})$ plaque larger than $6.0 \mathrm{~mm}^{3}$ was significantly correlated with culprit lesions in patients with $\mathrm{ACS}{ }^{16)}$

However, no study thus far has evaluated the correlation between the volume of such a low density $(<30 \mathrm{HU})$ plaque core and the IVUS-derived plaque components. In the present study, based on plaque quantification and using a more complex CCTA and IVUS assessment, we demonstrated that coronary plaques showing the presence of a low-density $(<30 \mathrm{HU})$ core $>6.0 \mathrm{~mm}^{3}$ on CCTA exhibited significantly larger IVUS components characteristic for unstable lesions than those without significant low-density cores (22.1 versus $17.9 \%, P=$ 0.001 , for necrotic core and 18.6 versus $15.8 \%, P=0.02$ for fibro-fatty tissue), although these plaques did not present significant differences in regard to other, non-vulnerable, VH-IVUS components (50.4 versus $54.2 \%, P=0.07$ for fibrotic tissue, and 8.7 versus $11.9, P=0.05$ for calcified tissue). Furthermore, LDC volumes determined by CCTA showed a high and statistically significant correlation with the percentage of the necrotic core at the site of the maximum area stenosis in culprit lesions $(r=0.7303, P<0.0001)$, indicating that the LDC core identified by CCTA could be in fact similar to the necrotic core of the plaque identified by VH-IVUS. However, there was also a good correlation observed with the percentage of fibro-fatty tissue by VH-IVUS in culprit lesions $(r=0.4928, P<0.0008)$, indicating some degree of overlap between vulnerable components in relation to their correspondence with the low-density plaque core on CCTA.

We also found that a low-density $(<30 \mathrm{HU})$ atheroma larger than $6.0 \mathrm{~mm}^{3}$ was present in a significantly greater extent in culprit lesions than in non-culprit ones (18.4 versus 6.5 $\left.\mathrm{mm}^{3}, P<0.001\right)$, suggesting that this could represent a new marker of vulnerability, similar to the other well-established ones.

Our study suggests that a low-attenuation plaque component with a CT density lower than $30 \mathrm{HU}$ is strongly associated with unstable plaques and therefore with an increased risk for plaque rupture, this finding being concordant with other previous studies. However, our study goes a step further and demonstrates that a critical core of $6.0 \mathrm{~mm}^{3}$ with low density $(<30$ $\mathrm{HU}$ ) by CCTA correlates well with the necrotic core of the plaque, as determined by IVUS, and therefore could be used to identify higher risks associated with the unstable plaques.

Interestingly, culprit plaques without a significant LDC did not present a VH-IVUS composition different from nonculprit ones, although their composition was significantly different from that of LDC-positive culprit plaques. This finding suggests that a LDC could represent a very sensitive marker for characterizing plaque vulnerability, as it can differentiate not only the culprit plaques from non-culprit ones but also culprit plaques with large necrotic cores from those with smaller cores.

Conclusions: Our analysis of plaque morphology using VHIVUS and CCTA as well as plaque quantification showed that the presence of a low-density $(<30 \mathrm{HU})$ core within the culprit lesions of patients with ACS represented a marker of vulnerability that correlated well with other CCTA and IVUS-derived features of vulnerability, especially with the necrotic core of the plaque.

The amount of low-density atheroma within the plaque, as determined with CCTA (using a density of $30 \mathrm{UH}$ and a volume of $6.0 \mathrm{~mm}^{3}$ as cutoff points for identifying low density atheroma) correlated well with VH-IVUS-derived markers of vulnerable plaques. Therefore, the identification of a significant low-density core within a coronary plaque on CCTA may provide a method to predict the risk associated with coronary lesions via a noninvasive method.

Given the current necessity for a cost-effective approach and justification of expensive imaging tests, together with the high degree of accuracy of information provided by CCTA using non-invasive plaque quantification, CCTA could in the future replace invasive techniques such as IVUS or optical coherence tomography for the complex evaluation of intracoronary plaque vulnerability.

\section{REFERENCES}

1. Falk E, Shah PK, Fuster V. Coronary plaque disruption. Circulation 1995; 92: 657-71. (Review)

2. Aziz K, Berger K, Claycombe K, Huang R, Patel R, Abela GS. Noninvasive detection and localization of vulnerable plaque and arterial thrombosis with computed tomography angiography/positron emission tomography. Circulation 2008; 117: 2061-70.

3. Virmani R, Burke AP, Farb A, Kolodgie FD. Pathology of the unstable plaque. Prog Cardiovasc Dis 2002; 44: 349-56. (Review)

4. Choy SY, Mintz GS. What have we learned about plaque rupture in acute coronary syndromes? Curr Cardiol Rep 2010; 12: 338-43. (Review) 
5. van Velzen JE, de Graaf FR, de Graaf MA, et al. Comprehensive assessment of spotty calcifications on computed tomography angiography: comparison to plaque characteristics on intravascular ultrasound with radiofrequency backscatter analysis. J Nucl Cardiol 2011; 18: 893-903.

6. Puri R, Worthley MI, Nicholls SJ. Intravascular imaging of vulnerable coronary plaque: current and future concepts. Nat Rev Cardiol 2011; 8: 131-9. (Review)

7. Calvert PA, Obaid DR, O'Sullivan M, et al. Association between IVUS findings and adverse outcomes in patients with coronary artery disease: the VIVA (VH-IVUS in Vulnerable Atherosclerosis) Study. JACC Cardiovasc Imaging 2011; 4: 894-901.

8. Boogers MJ, Broersen A, van Velzen JE, et al. Automated quantification of coronary plaque with computed tomography: comparison with intravascular ultrasound using a dedicated registration algorithm for fusion-based quantification. Eur Heart J 2012; 33: 1007-16.

9. Choi YH, Hong YJ, Park IH, et al. Relationship between coronary artery calcium score by multidetector computed tomography and plaque components by virtual histology intravascular ultrasound. J Korean Med Sci 2011; 26: 1052-60.

10. van Velzen JE, de Graaf FR, Jukema JW, et al. Comparison of the relation between the calcium score and plaque characteristics in patients with acute coronary syndrome versus patients with stable coronary artery disease, assessed by computed tomography angiography and virtual histology intravascular ultrasound. Am J Cardiol 2011; 108: 658-64.

11. Achenbach S, Raggi P. Imaging of coronary atherosclerosis by computed tomography. Eur Heart J 2010; 31: 1442-8. (Review)

12. Miller JM, Rochitte CE, Dewey M, et al. Diagnostic performance of coronary angiography by 64-row CT. N Engl J Med 2008; 359: 2324-36.

13. Hamon M, Morello R, Riddell JW, Hamon M. Coronary arteries: diagnostic performance of 16- versus 64-section spiral CT compared with invasive coronary angiography--meta-analysis. Radiology 2007; 245: 720-31.

14. Kitagawa T, Yamamoto H, Horiguchi J, et al. Characterization of noncalcified coronary plaques and identification of culprit lesions in patients with acute coronary syndromes by 64 -slice computed tomography. JACC Cardiovasc Imaging 2009; 2: 153-60.

15. Hoffmann U, Moselewski F, Nieman K, et al. Noninvasive assessment of plaque morphology and composition in culprit and stable lesions in acute coronary syndrome and stable lesions in stable angina by multidetector computed tomography. J Am Coll Cardiol 2006; 47: 1655-62.

16. Benedek T, Gyöngyösi M, Benedek I. Multislice computed tomographic coronary angiography for quantitative assessment of culprit lesions in acute coronary syndromes. Can J Cardiol 2013; 29: 364-71.
17. Hamm CW, Bassand JP, Agewall S, et al. ESC guidelines for the management of acute coronary syndromes in patients presenting without persistent ST-segment elevation: The Task Force for the management of acute coronary syndromes (ACS) in patients presenting without persistent ST-segment elevation of the European Society of Cardiology (ESC). Eur Heart J 2011; 32: 2999-3054.

18. Hausleiter J, Meyer T, Hermann F, et al. Estimated radiation dose associated with cardiac CT angiography. JAMA 2009; 301: 500-7.

19. Budoff MJ, Achenbach S, Blumenthal RS, et al. Assessment of coronary artery disease by cardiac computed tomography: a scientific statement from the American Heart Association Committee on Cardiovascular Imaging and Intervention, Council on Cardiovascular Radiology and Intervention, and Committee on Cardiac Imaging, Council on Clinical Cardiology. Circulation 2006; 114: 1761-91. (Review)

20. Kolodgie FD, Burke AP, Farb A, et al. The thin-cap fibroatheroma: a type of vulnerable plaque: the major precursor lesion to acute coronary syndromes. Curr Opin Cardiol 2001; 16: 285-92. (Review)

21. Fujii K, Hao H, Ohyanagi M, Masuyama T. Intracoronary imaging for detecting vulnerable plaque. Circ J 2013; 77: 588-95. (Review)

22. Kubo T, Matsuo Y, Ino Y, et al. Optical coherence tomography analysis of attenuated plaques detected by intravascular ultrasound in patients with acute coronary syndromes. Cardiol Res Pract 2011; 2011: 687515

23. Kato M, Dote K, Sasaki S, et al. Presentations of acute coronary syndrome related to coronary lesion morphologies as assessed by intravascular ultrasound and optical coherence tomography. Int J Cardiol 2013; 165: 506-11.

24. Cordeiro MA, Lima JA. Atherosclerotic plaque characterization by multidetector row computed tomography angiography. J Am Coll Cardiol 2006; 47: C40-7. (Review)

25. Ozaki Y, Okumura M, Ismail TF, et al. Coronary CT angiographic characteristics of culprit lesions in acute coronary syndromes not related to plaque rupture as defined by optical coherence tomography and angioscopy. Eur Heart J 2011; 32: 2814-23.

26. Kröner ES, van Velzen JE, Boogers MJ, et al. Positive remodeling on coronary computed tomography as a marker for plaque vulnerability on virtual histology intravascular ultrasound. Am J Cardiol 2011; 107: 1725-9.

27. Motoyama S, Masayoshi S, Harigaya $\mathrm{H}$, et al. Computed tomographic angiography characteristics of atherosclerotic plaques subsequently resulting in acute coronary syndrome. J Am Coll Cardiol 2009; 54: 49-57.

28. Motoyama S, Kondo T, Sarai M, et al. Multislice computed tomographic characteristics of coronary lesions in acute coronary syndromes. J Am Coll Cardiol 2007; 50: 319-26. 